\title{
A fracture-based model of periodic-arrayed indentation for rock cutting
}

\author{
$\underline{\text { Yu Jun Xie }}{ }^{1, *}$, Bo Yang Xiang' and Xiao Zhi Hu${ }^{2 *}$ \\ ${ }^{1}$ Department of Mechanical Engineering, Liaoning Shihua University, Fushun, 113001, P. R. China. \\ ${ }^{2}$ School of Mechanical and Chemical Engineering, The University of Western Australia, Perth, WA 6009, Australia. \\ * Corresponding author: yjxie@Inpu.edu.cn, xiao.zhi.hu@uwa.edu.au.
}

\begin{abstract}
When an half-space elastic substrate is subject to indentation with rigid, flat-ended and periodic indenters, a periodic singular stress fields and K-dominant regions should arise adjacent to the indenter edges. The concept of indentation stress intensity factor $K_{\text {ind }}$ is introduced to describe the singular indentation stress field, which mathematically is very similar to that of a mode I crack. The singular indentation stress field is sufficient to induce cracking from a smooth crack-free surface, which can potentially play a significant role in damage analysis of rock cutting. In the present article, a fracture-based model is proposed for rock breakage by using an energy-based method. The indentation stress intensity factor $\mathrm{K}_{\text {ind }}$ and indentation cracking equation for rock cutting model have been formulized.
\end{abstract}

Keywords Fracture, Contact mechanics, Indentation stress intensity factors, Indentation.

\section{Introduction}

A rock-cutting tool schematically illustrated in Fig.1 can be modeled by periodic rigid and flat-tipped indenters. It is noted from the work [1-4] that a mixed-mode singular stress field exists in an incompressible substrate at the sliding contact edge of a rigid flat-ended indenter pressing down onto the substrate. Subsequently, the damage in a form of the micro-crack initiation on the contact surface will be induced by such a singular stress field, which can contribute substantially to rock cutting. In the present work, a fracture-based method similar to the classical fracture mechanics is proposed to formularize the cracking or indentation damage induced by the singular indentation stress field next to the corners of the periodic indenters.

\section{Periodic indentation configurations}

The modeling of rock cutting problem to be investigated is illustrated in Fig.1. The periodic indenters is pressed on to the surface of half plane substrate, which occupies the region $0<x_{2}<h<+\infty,-\infty<x_{1}<+\infty$ and is constrained to deform in plane strain normal to the $x_{1}-x_{2}$ plane. The substrate is supposed to be elastically isotropic, with Young's modulus $E$ and Poisson's ratio $\mu$. The periodic arrayed indenters is supposed to be rigid, with contact width $2 l$ and center-to-center contact spacing $2 t$. The calculations for indentation stress intensity factors are carried out based on one of the periodic Cell 1 and Cell 2 that lies between $-t / 2<x_{1}<t / 2$. Two limiting cases of friction are considered: (i) perfect smooth sliding between the indenter and the substrate; and (ii) small frictional sliding contact between the indenter and the substrate.

\section{Asymptotic stress field in sliding contact}

\subsection{Boundary Condition}

A typical fretting contact problem of a rigid flat-ended indenter with half width a, sliding on a homogeneous, isotropic, elastic body in half plane is shown in Fig.2. The Cartesian coordinates $\left(x_{1}\right.$, $\left.x_{2}\right)$, and the polar coordinates $(r, \theta)$, both with the origin at the left edge of the indenter, are selected. Normal force $N$ and tangential force $Q$ act on the indenter and the following normal and shear 
tractions along interface have been solved in closed form [4],

$$
p\left(x_{1}\right)=-\frac{N \sin \lambda \pi}{\pi}\left(2-\frac{x_{1}}{a}\right)^{\lambda-1}\left(\frac{x_{1}}{a}\right)^{-\lambda}
$$

and

$$
q\left(x_{1}\right)=f p\left(x_{1}\right)
$$

where $f$ is the coefficient of friction; $\lambda$ is determined by

$$
\tan \lambda \pi=\frac{2(1-\mu)}{f(1-2 \mu)}, \quad 0<\lambda<1 .
$$

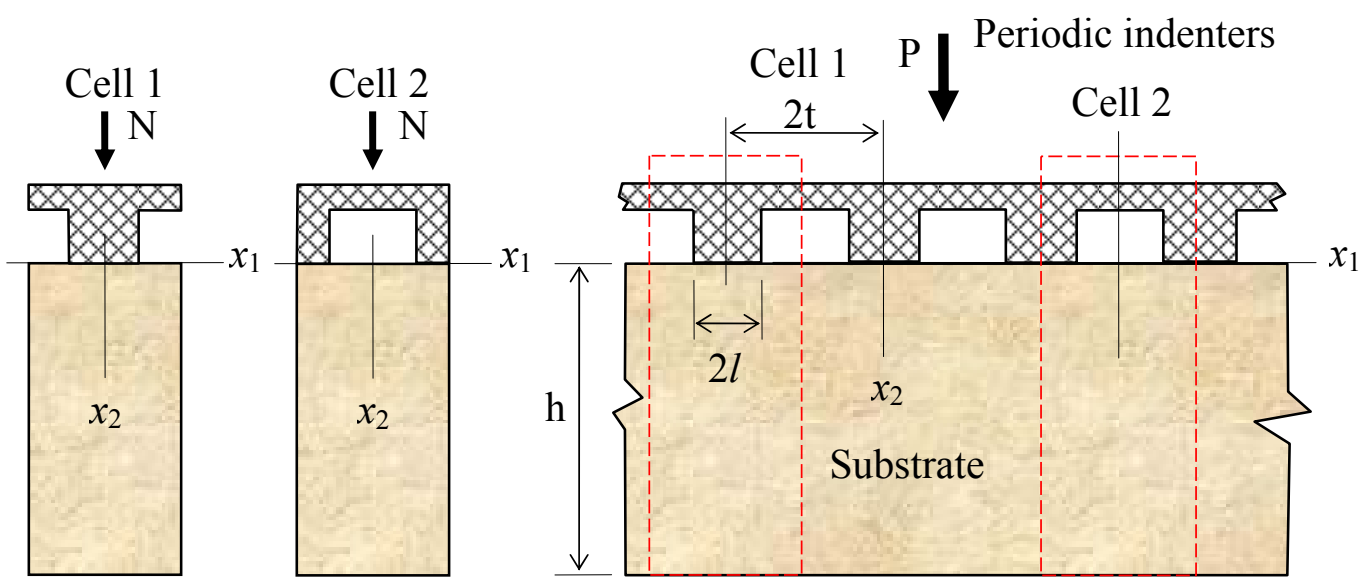

Fig. 1 The two-dimensional indentation model for rock cutting with $n$ periodic indentaters.

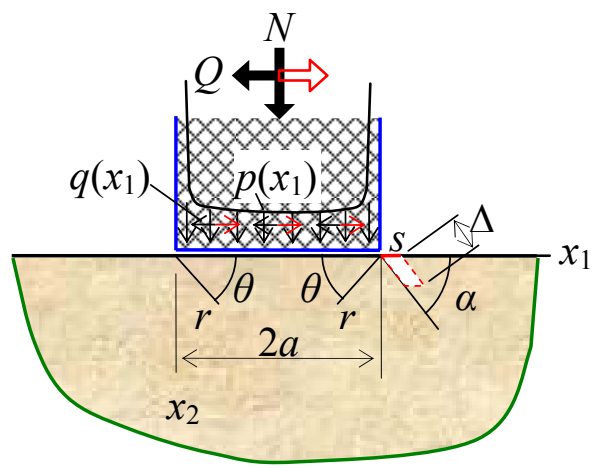

Fig.2. Indentation configuration, integral path $s \rightarrow 0$ and $\Delta \rightarrow 0$.

Eq.(1) shows that the stress state near the indenter corner may vary in the form

$$
\sigma_{i j}=\left\{\begin{array}{ll}
0\left(r^{\lambda-1}\right), & x_{1}=2 a \\
0\left(r^{-\lambda}\right), & x_{1}=0
\end{array} \quad \text { as } r \rightarrow 0 .\right.
$$

For special cases either with $\mu=0.5$ or $f=0$, Eq.(3) leads to $\lambda=0.5$, showing the same order of stress singularity as that for a sharp crack tip.

For $\mu=0.5$, the substrate becomes incompressible. The asymptotic stress boundary conditions of the substrate in the contact area next to the left and right corners then become

$$
\left.\sigma_{22}\right|_{\theta=0}=-\frac{N}{\pi \sqrt{2 a r}}
$$

and 


$$
\left.\sigma_{21}\right|_{\theta=0}=\frac{f N}{\pi \sqrt{2 a r}}
$$

for the two cases of $\mu=0.5$ and $f=0$.

\subsection{Singular stress fields due to the normal and tangential loads}

The singular stress field at the sharp edge of the contact between a rigid flat-ended indenter and substrate is known from the asymptotic contact analyses of and Nadai [4]. Using the polar coordinates $(r, \theta)$, Fig.2, the stresses at the left corner can be found as follows due to the normal load:

$$
\left(\begin{array}{l}
\sigma_{r r}^{I} \\
\sigma_{\theta \theta}^{I} \\
\sigma_{r \theta}^{I}
\end{array}\right)=-\frac{K_{I-i n d}}{\sqrt{2 \pi r}}\left(\begin{array}{l}
\cos \frac{\theta}{2}\left(1+\sin ^{2} \frac{\theta}{2}\right) \\
\cos ^{3} \frac{\theta}{2} \\
\sin \frac{\theta}{2} \cos ^{2} \frac{\theta}{2}
\end{array}\right) .
$$

This expression indicates that the stress state for indentation is a "negative" Mode-I singular stress field for cracked solids, where

$$
K_{I-i n d}=\frac{N}{\sqrt{\pi a}},
$$

which defines actually an indentation stress intensity factor. The familiar Mode-I singular stress field is obtained by removing the negative sign and changing $K_{\text {I-ind }}$ into $K_{\mathrm{I}}$ for cracked solids with mode-I loads. Only difference between tensile mode-I stress field and indentation stress field is sign "-" in their equations.

Nadai [4] gave also the asymptotic stress field due to the tangential load as

where

$$
\left(\begin{array}{l}
\sigma_{r r}^{I I} \\
\sigma_{\theta \theta}^{I I} \\
\sigma_{r \theta}^{I I}
\end{array}\right)=\frac{K_{I I-i n d}}{\sqrt{2 \pi r}}\left(\begin{array}{l}
\sin \frac{\theta}{2}\left(1-3 \sin ^{2} \frac{\theta}{2}\right) \\
-3 \sin \frac{\theta}{2} \cos ^{2} \frac{\theta}{2} \\
\cos \frac{\theta}{2}\left(1-3 \sin ^{2} \frac{\theta}{2}\right)
\end{array}\right),
$$

$$
K_{\text {II-ind }}=f K_{I-i n d} \text {. }
$$

Actually, the Eq.(9) is identical to the classical Mode-II singular stress fields when $K_{\text {II-ind }}=K_{\text {II }}$.

\subsection{Characters of the stress fields}

It is clear from the above discussion that the asymptotic stress field, $\sigma_{i j}=\sigma_{i j}^{I}+\sigma_{i j}^{I I}$, induced by the sliding contact is a typical mixed-Mode I-II singular stress field for incompressible substrates and friction free. This singular stress field is responsible for surface crack initiation on the crack-free surface of the substrate at the contact edge. This finding is significant as it shows that singularity and distribution of the stress field induced by surface contact of a flat-ended indenter are identical to those of a mixed-mode crack. As a result, the concepts of stress intensity factor and fracture toughness can now be introduced unambiguously into contact mechanics and associated contact damage. Therefore, Eqs.(7)-(10) represent an important advance by defining the indentation stress

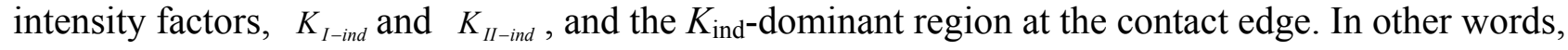
the fracture mechanics theory, such as the Griffith's criterion, is applicable in the case of the boundary fracture induced by the sliding contact. It should be pointed out that for finite boundary contact problems, Eqs.(7), (9) and (10) are still effective, for which case the $K_{I-i n d}$ should be 
solved by the concerned method.

\section{Conservation integral}

Based on the two-dimensional conservation law, for a closed integration path without any crack and cavity in it, the following integrals will vanish [5-8].

$$
J_{j}=\int_{s}\left(w n_{j}-T_{i} u_{i, j}\right) d s .
$$

Eq.(11) has two components, $J_{1}$ and $J_{2} . J_{1}$ is so-called J-integral. They can all be used to calculate the stress intensity factors for the cracked elastic bodies. In the following section some key steps to estimate the SIF for the indentation have been developed based on the $J_{2}$-integral.

\section{Stress intensity factors for periodic indentations}

As the definition of the half plane substrate, i.e., $0<x_{2}<h<+\infty,-\infty<x_{1}<+\infty$, any plane $x_{1}=k t, k=0,1,2 \ldots$ is the symmetrical plane. Then, the Cell 1 shown in Fig. 1 is considered for the calculation of the stress intensity factors for periodic indentations. Note that there are two singular stress fields next to the two corners of every indenter for all cells.

Select a closed integration path $s_{\text {abcdefghija }}$ as shown in Fig.3 for Cell 1. From the Eq.(11), following result can be given[5-8].

$$
J_{2}=\oint_{s_{a b c d e g h i j a}}\left(w n_{2}-T_{i} u_{i, 2}\right) d s=0 .
$$

For the paths $s_{b c}$ and $s_{d e}$, because the symmetry, $T_{2}=0, n_{2}=0$ and $u_{1,2}=0$, and the following result can be found.

$$
J_{2}=\int_{s_{b c}}\left(w n_{2}-T_{i} u_{i, 2}\right) d s=\int_{s_{d e}}\left(w n_{2}-T_{i} u_{i, 2}\right) d s=0 .
$$

Let the $s_{g h}$ and $s_{i j}$ be straight lines; and $s_{f g}$ and $s_{j a}$ a quarter of a circle. If the $s_{i j a}$ and $s_{f g h}$ are within the K-dominant regions, we have [11-13]

$$
J_{2}=\int_{s_{g h}}\left(w n_{2}-T_{i} u_{i, 2}\right) d s=\int_{s_{i j}}\left(w n_{2}-T_{i} u_{i, 2}\right) d s=0
$$

and

$$
J_{2}=\int_{s_{s_{g}}}\left(w n_{2}-T_{i} u_{i, 2}\right) d s=\int_{s_{j a}}\left(w n_{2}-T_{i} u_{i, 2}\right) d s=\frac{\left(1-\mu^{2}\right) K_{I-i n d}^{2}}{2 \pi E} \text { (plane strain). }
$$

When the cross sectional area where the $s_{c d}$ located is relatively far from the contact zone, the following expression can be given by

$$
J_{2}=\int_{s_{c d}}\left(w n_{2}-T_{i} u_{i, 2}\right) d s=\bar{w}^{-}+N \widetilde{u}_{2,2}^{-}
$$

where the $\tilde{u}_{i}$ shows the displacement of neutral axis. This displacement can be given by elementary strength theory of materials [11-13]. $\bar{w}$ is the strain energy density per unit length of the cell. For the $s_{h i}$, we have

$$
J_{2}=\int_{s_{h i}}\left(w n_{2}-T_{i} u_{i, 2}\right) d s=-\left(\bar{w}^{+}+N \widetilde{u}_{2,2}^{+}\right) .
$$

Then, substituting Eqs.(13)-(17) to Eq.(12), it gives

$$
J_{2}=\frac{\left(1-\mu^{2}\right) K_{I-i n d}^{2}}{\pi E}+2 \int_{s_{a b}} w d s=\left(\bar{w}^{-}+N \widetilde{u}_{2,2}^{-}\right)-\left(\bar{w}^{+}+N \widetilde{u}_{2,2}^{+}\right)=\frac{N}{2}\left(\widetilde{u}_{2,2}^{-}-\widetilde{u}_{2,2}^{+}\right),
$$

which can be interpreted as the energy release per unit moving of boundary $s_{i a b}$ and $s_{e f h}$ in 
$x_{2}$-direction or the crack mouth widening energy release rate for cracked solids [11-13]. The axial strain $\tilde{u}_{2,2}^{-}, \tilde{u}_{2,2}^{+}$have been found[11-13]

$$
\tilde{u}_{2,2}^{-}=-\frac{N}{E A}
$$

and

$$
\widetilde{u}_{2,2}^{-}=-\int_{0}^{1} \frac{N d \xi}{E A\left[1-(1-l / t) \sqrt{1-\xi^{2}}\right]}=-\frac{N}{E A}\left(\frac{2}{(1-l / t) \sqrt{1-(1-l / t)^{2}}} \arctan \sqrt{\frac{2-l / t}{l / t}}-\frac{\pi}{2(1-l / t)}\right) .
$$

Because the free action of the substrate surface and $s_{a b}$ and $s_{e f}$ is out of the K-dominant regions, the integral in left-hand side of Eq. (18) is a small quantity, which can be neglected. Substituting Eqs.(19) and (20) into Eq.(18), it will yield

$$
K_{I-i n d}=\frac{N}{\sqrt{\pi t}} f\left(\frac{l}{t}\right)
$$

where the normalized stress intensity factor can be found as

$$
f\left(\frac{l}{t}\right)=\frac{\pi}{2}\left\{\frac{1}{1-\mu^{2}}\left[\frac{2}{(1-l / t) \sqrt{1-(1-l / t)^{2}}} \arctan \sqrt{\frac{2-l / t}{l / t}}-\frac{\pi}{2(1-l / t)}-1\right]\right\}^{1 / 2} .
$$

Eq.(22) varying with perimeter $l / t$ is given in Fig.4.
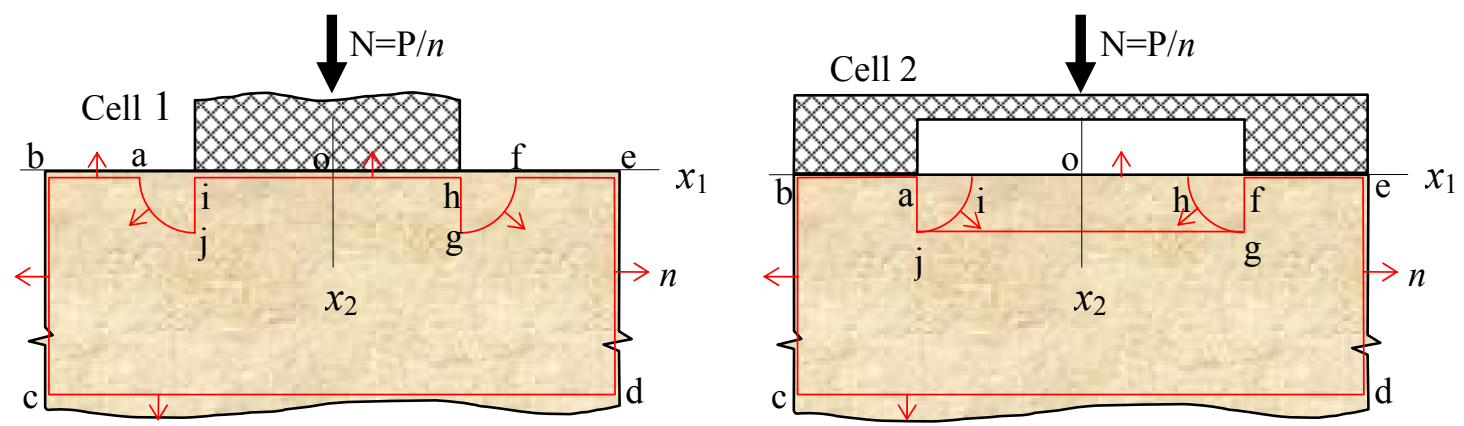

Fig.3. Contours of integration for the Cell 1 and Cell 2.

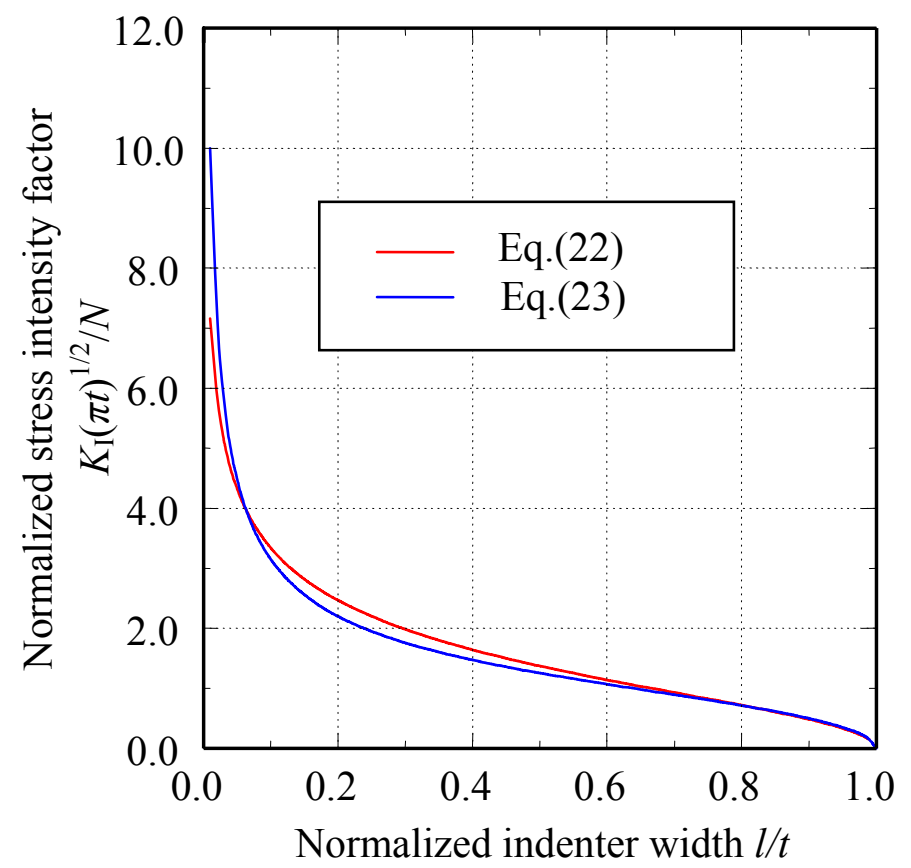

Fig.4. the scheme of the normalized stress intensity factor. 


\section{An indirect technique on the periodic indentation}

In above sections, a simple and direct method is proposed to estimate the indentation stress intensity factor for singular stress field induced by indentation. In this section, an alternative and indirect technique is considered.

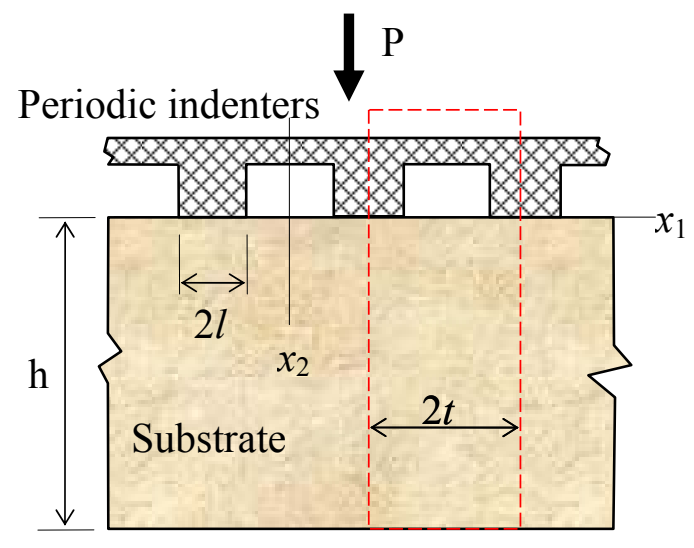

(a)

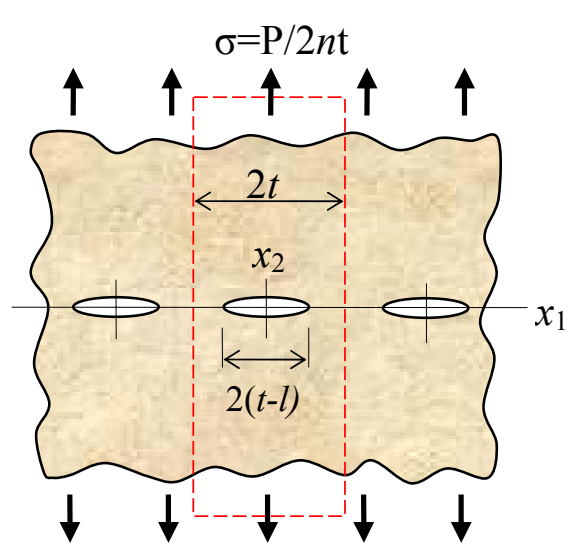

(b)

Fig.5. Periodic indentation and related periodic crack model. (a) Periodic indentation; (b) Related crack model.

For any Mode-I indentation, the related crack model can be found[9]. For various crack configurations, the stress intensity factors had been found and collected in the handbooks such as [14]. Hence, a simple and indirect technique of the crack analogue may be used to solve the indentation stress intensity factor for the Mode-I indentation based on the existing literature for the crack analysis. As a main research object in present work, i.e., periodic indentation, the related crack model can be given in Fig.5(b). For such a periodic array of collinear cracks, the exact solution of the stress intensity factor can be found [14].

$$
K_{I-i n d}=\frac{N}{\sqrt{\pi t}}\left\{\frac{\pi}{2} \tan \left[\frac{\pi}{2}\left(1-\frac{l}{t}\right)\right]\right\}^{1 / 2} .
$$

It varying with the normalized indenter width $l / t$ can be found in Fig. 4 .

\section{Boundary cracking in complete sliding contact}

\subsection{Energy release rate for boundary cracking}

From the geometrical point of view, boundary crack initiation can always be defined as a boundary movement in some direction, with the limit $s \rightarrow 0$ taken and the notch-like boundary becomes a crack, as shown in Fig.2. Then, the energy release rate of boundary cracking can be defined as[10-13]

where

$$
G=\left.\left(J_{1}\right)\right|_{s \rightarrow 0} \cos \alpha+\left.\left(J_{2}\right)\right|_{s \rightarrow 0} \sin \alpha,
$$

$$
\left.\left(J_{1}\right)\right|_{s \rightarrow 0}=\lim _{s \rightarrow 0} \int_{s} w n_{1} d s=\int_{s_{\text {in }}}\left(w n_{1}-T_{i} u_{i, 1}\right) d s
$$

and

$$
\left.\left(J_{2}\right)\right|_{s \rightarrow 0}=\lim _{s \rightarrow 0} \int_{s} w n_{2} d s=\int_{s_{i n}}\left(w n_{2}-T_{i} u_{i, 2}\right) d s .
$$

The integration path $s_{\text {in }}$ in Eqs.(25) and (26) is any integration path within the substrate, and $s_{\text {in }}+s$ 
form a closed integral loop. The $\left.\left(J_{1}\right)\right|_{s \rightarrow 0}$ denotes the driving force of boundary cracking in direction $x_{1}$ when the limit taken exists, or the energy release rate with unit boundary movement $s$ in direction $x_{1} ;\left.\left(J_{2}\right)\right|_{s \rightarrow 0}$ denotes the driving force in direction $x_{2}$, or the energy release rate with unit boundary movement $\mathrm{s}$ in direction $x_{2}$.

For a homogenous and isotropic substrate Griffith's criterion [15] states that the crack will extend when the critical value $G_{\max }$ is reached $[15,16]$

$$
G_{\max }=G_{c} .
$$

For a standard cracked specimen subjected to Mode-I loading, $G_{\max }=J_{1}$ and $G_{c}$ can be calibrated as

$$
G_{c}=J_{I C}=K_{I C}^{2}\left(1-\mu^{2}\right) / E,
$$

where $\mathrm{K}_{\mathrm{IC}}$ is the Mode-I fracture toughness. However, in the present case of surface crack initiation, a mixed-mode condition is expected as the singular stress field is generated by the sliding contact with the rigid flat-ended indenter.

\subsection{Critical cracking angle and critical load.}

Firstly, $\left.\left(J_{1}\right)\right|_{s_{o b} \rightarrow 0}$ can be found. Let $s=s_{o b}$ in Eq.(25) as shown in Figs.2 and 6 adjacent to the right

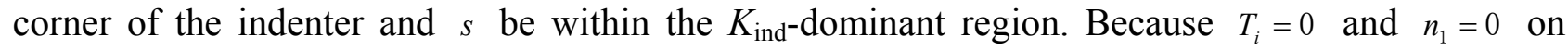
the integration path $s_{o b}$, the energy-based driving force for boundary cracking in $x_{1}$ direction in this case can be found as

$$
\left.\left(J_{1}\right)\right|_{s_{o b} \rightarrow 0}=\lim _{s_{o b} \rightarrow 0} \int_{s_{o b}} w n_{1} d s=0 .
$$

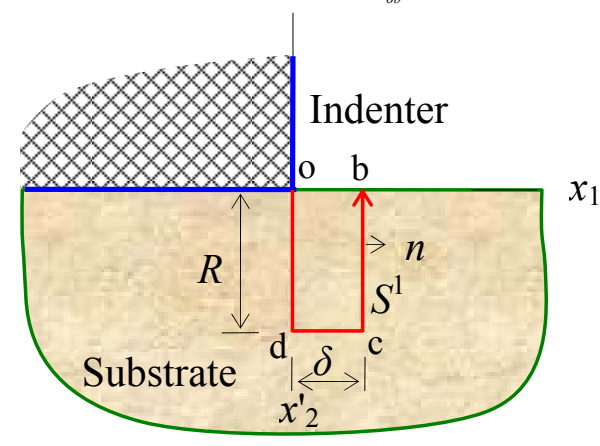

Fig.6. Integration path adjacent to punch corner within the $\mathrm{K}_{\text {ind }}$-dominant region for $J_{2}$.

Next step is to calculate $\left.J_{2}\right|_{s_{o b} \rightarrow 0}$. As shown in Figs.2 and 6, let $s=s_{o b}, s_{i n}=S_{\text {odcb }}^{1}$ in Eq.(26) and take the limit $s_{o b} \rightarrow 0, \delta / R \rightarrow 0$ and $R \rightarrow 0$, which means that the $s_{i n}=S_{o d c b}^{1}$ is within the

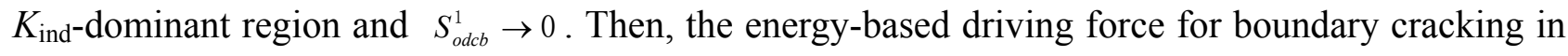
$x_{2}$ direction becomes $[10,16]$

$$
\left.\left(J_{2}\right)\right|_{s_{o b} \rightarrow 0}=\lim _{S_{a b} \rightarrow 0} \int_{S_{o b}} w n_{2} d s=-\frac{4\left(1-\mu^{2}\right)}{E} \lim _{\substack{\delta / R \rightarrow 0 \\ R \rightarrow 0}} \int_{S_{o d c h}^{2}} \operatorname{Im}\left(\Phi^{2}+\Phi \bar{\Phi}-z \bar{\Phi} \bar{\Phi}-\bar{\Phi} \bar{\Psi}\right) n_{1} d s,
$$

where

$$
\Phi(z)=\frac{K_{I}-K_{I I} i}{2 \sqrt{2 \pi z}}, \Psi(z)=\frac{K_{I}+3 K_{I I} i}{4 \sqrt{2 \pi z}},
$$

in which $K_{I}=-K_{I-\text { ind }}$ and $K_{I I}=K_{I I-i n d}$ for indentation singular stress fields. From Eqs.(30) and (31), it can be readily obtained that 


$$
\left.\left(J_{2}\right)\right|_{s \rightarrow 0}=\frac{\left(1-\mu^{2}\right) K_{I-i n d}^{2}}{2 \pi E}\left(1+\pi f-f^{2}\right) \text {, for the plane strain condition. }
$$

From Eqs.(24), (29) and (32), the total energy release rate of boundary cracking induced by the sliding contact at any angle $\alpha$ can be found as

$$
G=\left.\left(J_{1}\right)\right|_{s_{o b} \rightarrow 0} \cos \alpha+\left.\left(J_{2}\right)\right|_{s_{o b} \rightarrow 0} \sin \alpha=\frac{\left(1-\mu^{2}\right) K_{I-i n d}^{2}}{2 \pi E}\left(1+\pi f-f^{2}\right) \sin \alpha .
$$

Setting $\frac{d G}{d \alpha}=0$, i.e., $\cos \alpha_{c}=0$, we have

$$
\alpha_{c}=\frac{\pi}{2},
$$

where $\alpha_{c}$ is the critical cracking angles, which is vertical to the contact boundary.

Cracking occurs when $G$ reaches its critical or maximum value. The critical or maximum energy-based driving force for boundary cracking can then be solved from Eq. (33), i.e.

$$
G_{\max }=\frac{\left(1-\mu^{2}\right) K_{I-i n d}^{2}}{2 \pi E}\left(1+\pi f-f^{2}\right) .
$$

From the Eqs.(27) and (35), the critical condition of substrate boundary cracking beneath the contact surface can be found as

$$
G_{\max }=\frac{\left(1-\mu^{2}\right) K_{I-i n d}^{2}}{2 \pi E}\left(1+\pi f-f^{2}\right)=G_{I C} .
$$

Finally, the normalized critical load $\mathrm{P}$ can be expressed as

$$
\frac{P_{c}}{n \sqrt{\pi t} K_{I C}}=\frac{2}{\left(1+\pi f-f^{2}\right)^{1 / 2} \tan ^{1 / 2}[0.5 \pi(1-l / t)]}
$$

for a given indenter size $2 l$ from Eqs.(23), (28) and (36). Fig.7 shows the effect of perimeter $l / t$ on the normalized critical loads.

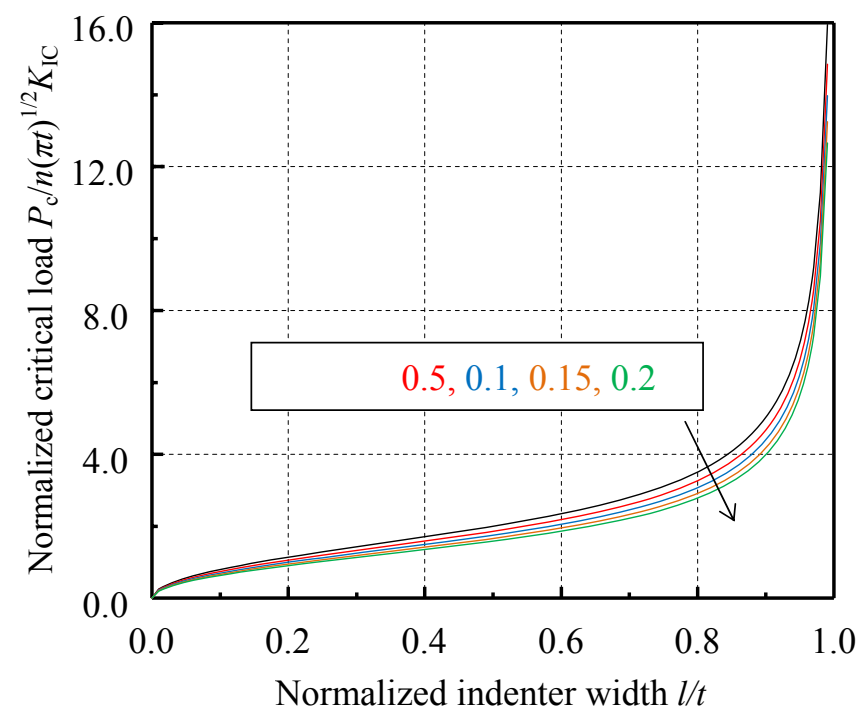

Fig.7. The effect of the normalized indenter width on the normalized critical load.

\section{Conclusions}

A fracture model of periodic indentation for rock cutting is proposed and formulated by using an energy-based method. The present approach specifies the contribution of indentation on the rock 
cutting and provides a closed form solution for the energy release rate of mixed-mode boundary cracking as determined by the mixed mode singular stress field induced by the sliding contact. The

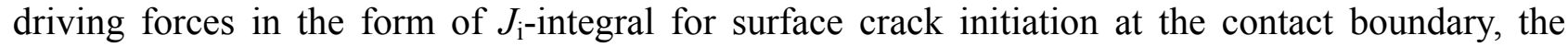
critical energy release rate and the critical load have been analytically determined. The critical cracking angles, critical loads have also been derived.

The present study shows that the frictional coefficient between the indenter and substrate uniquely characterizes the asymptotic singular stress field, and hence is considered as the primary parameter to determine the critical load of the indenter under the condition of slip near the edge of contact. The findings in this study may be helpful for establishing the damage mechanisms in the complex process of rock cutting by tools with the flat-tipped teeth.

\section{Acknowledgement}

This work was supported by National Natural Science Foundation of China (Grant Nos. 50771052 and 50971068, 11272141), Gledden Senior Fellowship from the University of Western Australia, and Natural Science Foundation of Liaoning (Grant Nos. LS2010100 and 20102129).

\section{References}

[1.] E. Giannakopoulos, T. C. Lindley, and S. Suresh, Aspects of connections and life-prediction methodology for fretting-fatigue. Acta Mater, 46 (1998) 2955-2968.

[2.] E. Giannakopoulos, T. A. Venkatesh, T. C. Lindley and S. Suresh, The role of adhesion in contact fatigue. Acta Mater, 47 (1999) 4653-4664.

[3.] B. Yang and S. Mall, On crack initiation mechanism in fretting fatigue. ASME J. Appl. Mech, 68 (2001) 76-80.

[4.] A. I. Nadai, Theory of flow and fracture of solids, McGraw-Hill, New York, 1963.

[5.] J. D. Eshelby, The force on an elastic singularity. Phil. Trans. Roy. Soc. London Ser. A, 244 (1951) 87-112.

[6.] G. C. Sih. Inelastic behaviour of solids. In: Sih GC editor. Dynamic aspects of crack aspects of crack propagation, New York: Mc-Graw-Hill Book, Co; 1969, p.607-639.

[7.] J. R. Rice, A path-independent integral and the approximate analysis of strain concentrated by notches and cracks. ASME J. Appl. Mech, 35 (1968) 379-386.

[8.] B. Budiansky and J. R. Rice, Conservation laws and energy-release rates. ASME J. Appl. Mech, 40 (1973) 201-203.

[9.] Y. J. Xie and D. A. Hills, Quasibrittle fracture beneath a flat bearing surface. Eng. Fract. Mech, 75 (2008) 1223-1230.

[10.]Y. J. Xie, X. Z. Hu, and X. H. Wang, Frictional contact induced crack initiation in incompressible substrate. Eng Fract Mech. 2011; 78: 2947-2956.

[11.] Y. J. Xie, A Theory on Cracked Pipe. Int. J. Press. Vessel. and Pip, 75 (1998) 865-869.

[12.] Y. J. Xie, X. Zhang and X. H. Wang, An exact method on penny-shaped cracked homogeneous and composite cylinders. Int. J. Solid. and Struct, 38 (2001) 6953-6963.

[13.]Y. J. Xie, An analytical method on circumferential periodic cracked pipes and shells. Int. J. Solid. and Struct, 37 (2000) 5189-5201.

[14.]H. Tada, P. C. Paris and G. R. Irwin, The stress analysis of cracks handbook (2nd Ed.), Paris Productions, Inc., St. Louis, 1985.

[15.]A. Griffith. The Phenomena of rupture and flow in solids. Phil. Trans. Roy. Soc. London Ser. A, 221 (1921) 163-198.

[16.] G. P. Cherepanov, Mechanics of brittle fracture, McGraw-Hill International Book Co, New York, 1979. 